\title{
Development of Molecularly-Imprinted Fluorescent Fabric Based Sensor System for the Recognition of a Model Azo-Dye Molecule ${ }^{+}$
}

\author{
Murat Barsbay *, Masoomeh Mehrnia \\ † Presented at the 2nd International Online-Conference on Nanomaterials, 15-30 November 2020; Available \\ online: https://iocn2020.sciforum.net/.
}

Published: 15 November 2020

\begin{abstract}
The main purpose of this study is to develop a sensitive and portable fluorescent sensor system with a fast response time to be used to detect a target analyte. An azo-dye (Basic Red 9) was selected as a model target molecule. The luminescence section of the fluorescent sensor system is composed of UpConverting NanoParticles (UCNP). These luminescent particles were covered by a "recognition element" which is a molecularly imprinted polymer (MIP) shell. MIP shell was synthesized by applying a controlled polymerization technique, namely RAFT polymerization. The MIP covered UCNP core-shell particles (MIP@UCNP) were then covalently attached on the surface of non-woven polyethylene/polypropylene (PE/PP) fabrics via azide-alkyne click coupling reaction. Reusable target molecule-recognizing fluorescent fabrics were therefore prepared. The materials attained in each synthesis step were characterized by TEM, SEM, SEM-EDX, XPS, FTIR and XRD methods. The binding performances were investigated by fluorescence spectroscopy, which yielded a LoD at a few-ppb level. The sensor system developed is (1) more sensitive than similar ones thanks to the well-defined attitude of the MIP shell, (2) user-friendly, portable, practical and low-cost owing to the covalent attachment of nanoparticles on the surface of fabrics.
\end{abstract}

Keywords: upconverting nanoparticles; lanthanides; molecularly imprinted polymers; RAFT polymerization; florescence sensor systems

\section{Introduction}

Upconverting nanoparticles (UCNPs) are an individual group of optical nanomaterials doped with lanthanide ions featuring an abundance of electronic transitions within the $4 \mathrm{f}$ electron shells. These nanoparticles can up-convert two or more lower-energy photons into one high-energy photon [1]. This unique anti-Stokes optical property has enabled a broad range of applications, spanning from background-free biological sensing and light-triggered drug delivery to solar energy harvesting and super-resolution microscopy [2-4]. UCNPs consist of rare earth elements (lanthanides) doped in a matrix [1,5-7]. UCNP's matrix is expected to be chemically stable and non-interfering structures. In addition to the matrix and the doped lanthanides, the parameters such as the size, shape, morphology, homogeneity, functionality and crystal structure of UCNPs are factors affecting the emission intensity and efficiency $[1,8-10]$. There are two forms of these crystals; the hexagonal $(\beta)$ and the cubic $(\alpha)$ phases $[4,9]$. Hexagonal phase, due to the increased symmetry, is chemically more stable and shows an order of magnitude higher upconversion efficiency compared to cubic phase. $\alpha$ -NaYF4 is a high-temperature metastable phase, while $\beta$-NaYF4 is a thermodynamically stable one. However, $\alpha$-NaYF4 as a kinetic product usually preferentially forms at the initial stage via the conventional wet synthetic routes. Generally, high temperature $\left(\geq 300{ }^{\circ} \mathrm{C}\right)$ and long aging time are required to overcome the high energy barrier for $\alpha$ to $\beta$ phase transformation on the kinetic opinion 
[4]. Modification of the surface of the nanoparticles has been one of the frequently used to increase and diversify the application of them [11-13]. Many different methods have been applied in the literature to modify the surfaces of UCNPs $[6,14,15]$. The applied methods are expected to have criteria such decreasing toxicity, exhibiting chemical stability, increasing surface functionality and reducing energy loss. Coating of the surfaces with the $\mathrm{SiO}_{2}$ shell is among the most frequently applied surface modifications, since it meets all these criteria [18-20]. The combination of UCNPs with a $\mathrm{SiO}_{2}$ shell has provided the way for nanoparticles to have an increasing use in many applications such as drug release, bio imaging, and sensors [6]. Molecularly imprinted polymers (MIP) are cross-linked structures that contain recognition regions specific to a particular as model or target molecule in their building $[16,17]$. The interaction between the target molecule and the polymeric shell occurs through interactions such as covalent bonding, non-covalent bonding or hydrogen bonding $[18,19]$. In the field of sensors, upconversion snanoparticles (UCNPs) and molecularly imprinted polymers (MIP) are of great interest. To date, there have been a few reports on the combination of UCNPs and MIP for sensing $[16,17,20]$.

In this study, the UCNPs doped hexagonal $\beta$-NaYF4 were prepared by optimized hydrothermal synthesis method. Three different methods have been applied to coat the UCNPs surfaces with MIPs. in the first method, UCNPs were directly coated with the MIP shell. In the characterization results obtained as a result of this method, it was showed that the UCNPs were not homogeneously covered with MIP. In the second method, it was aimed to cover the nanoparticles with MIP shell in the presence of a radically-forming photo-initiator (eosin-Y). This method consists of using the light generated by the visible or UV emissions of the UCNPs when excited with a laser $(980 \mathrm{~nm})$ light source to construct a thin polymer shell around the UCNPs by photopolymerization method [20,21]. According to this method, while the target molecule was not in the solution, the light emitted by the UCNPs that radiated in the visible region when they were excited at $980 \mathrm{~nm}$ triggered the radical production of the eosin-Y and the non-imprinted polymers (NIPs) were successfully formed around the UCNPs. However, when the experiments were repeated in the presence of the target molecule (BR9), the eosin-Y was not excited and the MIP could not be formed around the UCNPs because the target molecule absorbed the radiation produced by the UCNPs. In the third method, primarily UCNPs surfaces were coated with $\mathrm{SiO}_{2}$ using TEOS. Coating step with $\mathrm{SiO}_{2}$ has been optimized by trying both 4 different Stöber and Microemulsion methods. Following the determination of the appropriate method, the surfaces of the nanoparticles coated with $\mathrm{SiO}_{2}$ were functionalized with vinyl groups using triethoxyvinyl silane. Then leading surfaces which was functionalized with vinyl groups were coated with the MIP shell by RAFT polymerization. The parameters such as monomertarget molecule complex stability, [RAFT agent]/[thermal initiator] and [monomer]/[RAFT agent] molar ratios and reaction times were optimized. As a result of all the characterizations and optimizations, the method using the precursor surfaces functioning with vinyl groups has been determined as the most appropriate synthesis method. Following the synthesis of the UCNPs coated with MIP and NIP shells, the binding performance of these nanoparticles; incubation time, reusability, kinetic studies were investigated by considering parameters such as different target molecule concentrations, competitive and real sample medium. A highly selective and low detection limit $(0.7 \mathrm{ppb})$ molecularly imprinted fluorescent sensor platform was developed in this work. Unlike UCNPs based sensor studies in the literature, polymerization steps within the scope of the study were carried out in a controlled manner with RAFT polymerization and superior properties were obtained [22-24]. The method developed is important as it has the potential to adapt to many molecules, as well as the dye (BR9) selected as the model compound. 


\section{Experimental}

\subsection{Chemicals}

Ethylene glycol dimethacrylate (EGDMA), 4,4'-azobis-4-cyanopentaonic acid (ACPA), basic red 9 (BR9), oleic acid, sodium hydroxide $(\mathrm{NaOH})$, sodium fluoride $(\mathrm{NaF})$, yttrium nitrate hexahydrate $\left(\mathrm{Y}(\mathrm{NO} 3) 6 \mathrm{H}_{2} \mathrm{O}\right)$, ytterbium (III) nsitrate pentahydrate $\left(\mathrm{Yb}(\mathrm{NO} 3) 5 \mathrm{H}_{2} \mathrm{O}\right)$, erbium nitrate pentahydrate (Er(NO3) $5 \mathrm{H}_{2} \mathrm{O}$ ), cumyl dithiobenzoate (CDB), tetraethyl orthosilicate (TEOS), 2', 4'. When buying 5', 7'-tetrabromofluorescein (eosin Y), dimethyl sulfoxide (DMSO), triethylamine (TEA), triethoxyvinyl silane (TEVS), Triton X-100, cyclohexane, methanol were purchased from SigmaAldrich. Ethanol and methacrylic acid (MAA) were obtained from Merck.

\subsection{Synthesis of the UCNPS Doped Hexagonal $\beta$-NaYF4}

6 different methods have been applied to optimize UCNPs synthesis (supporting information) and 6th method was chosen as the protocol method for the synthesis of the UCNPs.

In a typical experiment, $1.2 \mathrm{~g} \mathrm{NaOH}, 20.0 \mathrm{~mL}$ oleic acid and $7.0 \mathrm{~mL}$ ethanol were mixed at room temperature until a white viscous solution was obtained. Then $5.0 \mathrm{~mL}$ of $\mathrm{H}_{2} \mathrm{O}$ and $8.0 \mathrm{~mL}$ of $1 \mathrm{M} \mathrm{NaF}$

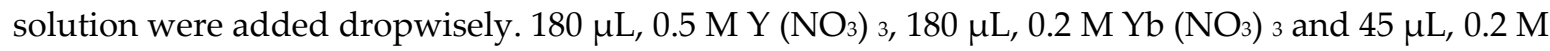
$\mathrm{Er}\left(\mathrm{NO}_{3}\right)_{3}$ were mixed in to the above solution and after $10 \mathrm{~min}$ the solution was transferred into a Teflon autoclave, which was tightly sealed and maintained at $210{ }^{\circ} \mathrm{C}$ for $20 \mathrm{~h}$. At the end of the reaction, the solution was centrifuged to remove UCNPs and the nanoparticles were washed with a mixture of ethanol: water [24].

\subsection{UV-Vis Spectrophotometry}

UV-vis Varian Cary100 spectrophotometer was used in selectivity studies. Measurements were performed between $190-800 \mathrm{~nm}$ wavelength at room temperature.

\subsection{Attenuated Total Reflectance Fourier Transform Infra-Red (ATR-FTIR)}

Spectroscopy FTIR spectra were recorded in attenuated total reflection (ATR) mode by Perkin Elmer Spectrum One model spectrometer. Spectra were obtained by 32 scans with a $4 \mathrm{~cm}^{-1}$ resolution.

\subsection{X-Ray Photoelectron Spectroscopy (XPS)}

X-ray photoelectron experiments were performed using monochromatized Al K $\alpha$ X-ray source of Thermo spectrometer. The analysis of the elemental composition on the surface was carried out with 0-1000 eV binding energy, with $400 \mu \mathrm{m}$ X-rays spot size.

\subsection{Scanning Electron Microscopy (SEM)}

SEM analyzes were performed under vacuum and with the SEM (FEI, Quanta 200F, Lausanne, Switzerland) using the backscattered electron technique at an acceleration voltage of $15.00 \mathrm{kV}$.

\subsection{Transmission Electron Microscope (TEM)}

FULL analyzes of the samples were performed with FEI Brand Tecnai G2 F30 Model TEM.

\subsection{X-Ray Diffractometer (XRD)}

XRD analyzes of the samples were carried out in the range of $2 \theta=10^{\circ}-90^{\circ}$ and $8 \mathrm{rpm}$ for homogenous data collection using the PANalytical X'Pert Powder Diffractometer with CuK $\alpha$ radiation. 


\subsection{Fluorescence Spectroscopy}

As a result of the excitation of UCNPs at $980 \mathrm{~nm}$ wavelength with laser, the measurement of the emission absorbance (around $540 \mathrm{~nm}$ ) was performed with Perkin Elmer LS-55 Fluorescence Spectrophotometer.

\section{Results and Discussion}

\section{Synthesis of the UCNPs Doped $\beta-N a Y F_{4}$ Crystals}

6 different synthesis methods were performed and the most appropriate synthesis method was determined as a result of XRD, SEM, XPS, TEM and fluorescence spectroscopy analysis of UCNPs doped $\beta$-NaYF4 crystals. In choosing the most advantageous synthesis method, size distribution, morphology and the florescence intensity of nanoparticles are important parameters. The experimental details of the 6 methods which applied to optimize UCNPs synthesis are summarized in supporting information part.

According to the TEM and SEM analysis the UCNP's with homogeneous structure and size were synthesized (Figure 1). The results of SEM and TEM analysis were compared with the results in the related references. The analysis results obtained with X-ray diffraction (XRD) also were interpreted with the references (the reference with 16-0334 number in the database for the hexagonal phase and the reference with 13-7404 number for the cubic phase).

The XRD results, showed that the peak of NaF has decreased where the intensity of the other peaks belonging to the hexagonal phase has increased. The structure shows high observance with the hexagonal phase ( $\beta$ phase) and there is no alpha phase in the final product (Figure 1$)$. The XRD patterns indicated that the nanostructures obtained with 6th method consist of pure $\beta$-NaYF4 (Figure $1)$.

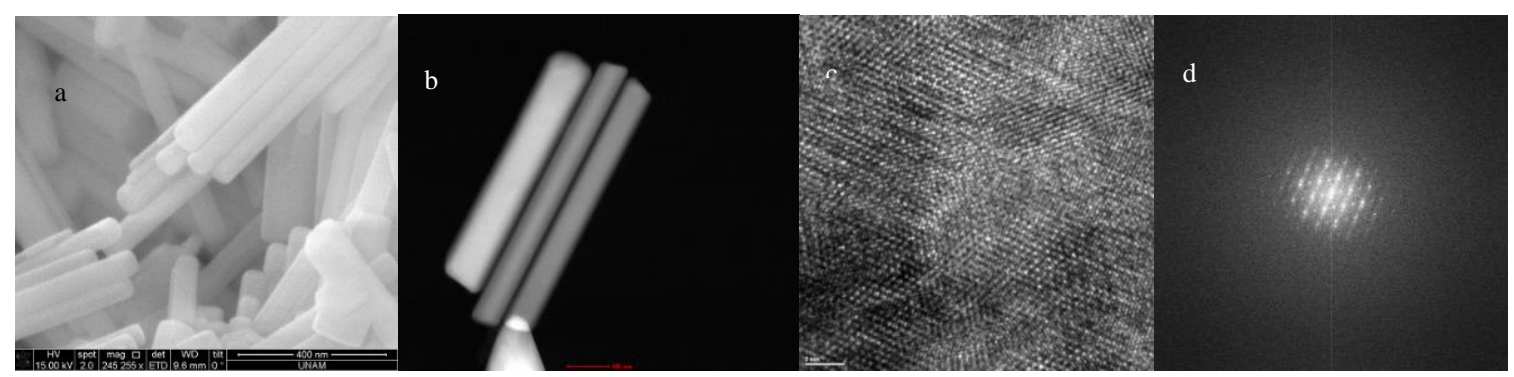

g)
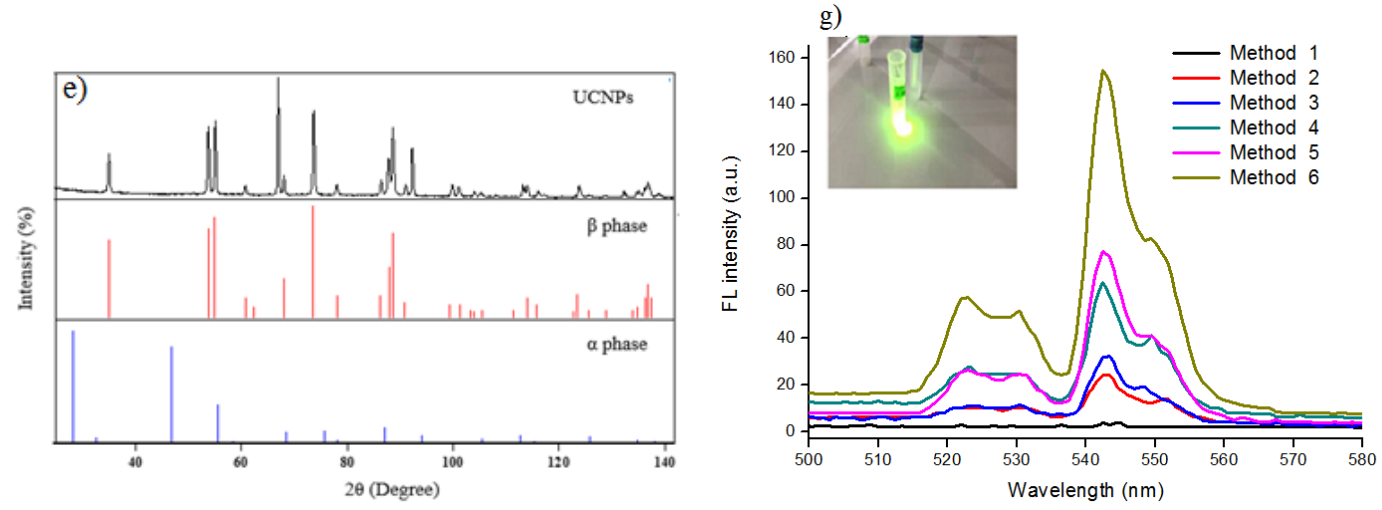

Figure 1. (a) SEM, (b) TEM, (c) High-Resolution TEM images, (d) TEM images Showing Hexagonal ( $\beta$ phase) of UCNPs (e) XRD results and XRD patterns of the UCNPs powders. (g) The maximum Fluorescence absorbance of UCNPs obtained as a result of each synthesis method in the range of 500$580 \mathrm{~nm}$ and typical photograph of the UCNPs under excitation.

Stretching peaks of the COO- groups at approximately $1547 \mathrm{~cm}^{-1}$ and $=\mathrm{C}-\mathrm{H}$ groups at $2900 \mathrm{~cm}^{-1}$ were seen in the unmodified UCNPs [25]. In the spectrum of UCNPs covered with $\mathrm{SiO}_{2}$, the peak of 
the $\mathrm{Si}-\mathrm{O}$ bond appearing at $1079 \mathrm{~cm}^{-1}$ resulting of $\mathrm{SiO}_{2}$ modification [17]. Following modification of $\mathrm{SiO}_{2}$ coated UCNPs with vinyl groups, the characteristic $\mathrm{C}=\mathrm{C}$ stress peak was detected at $1635 \mathrm{~cm}^{-1}$ [19]. The wide peak observed around $3400 \mathrm{~cm}^{-1}$ originates from the $-\mathrm{OH}$ groups and the intensity of this peak increases after the UCNPs surface was coated with the recognizing MIP shell [4]. In the spectrum of MIP @ UCNPs, the peaks of the C-H stretch at $2900 \mathrm{~cm}^{-1}$, the carbonyl observed at 1703 $\mathrm{cm}^{-1}$ and the ether (C-O-C) groups at $1163 \mathrm{~cm}^{-1}$ indicates that UCNPs are successfully covered with the MIP shell (Figure 2) [17].

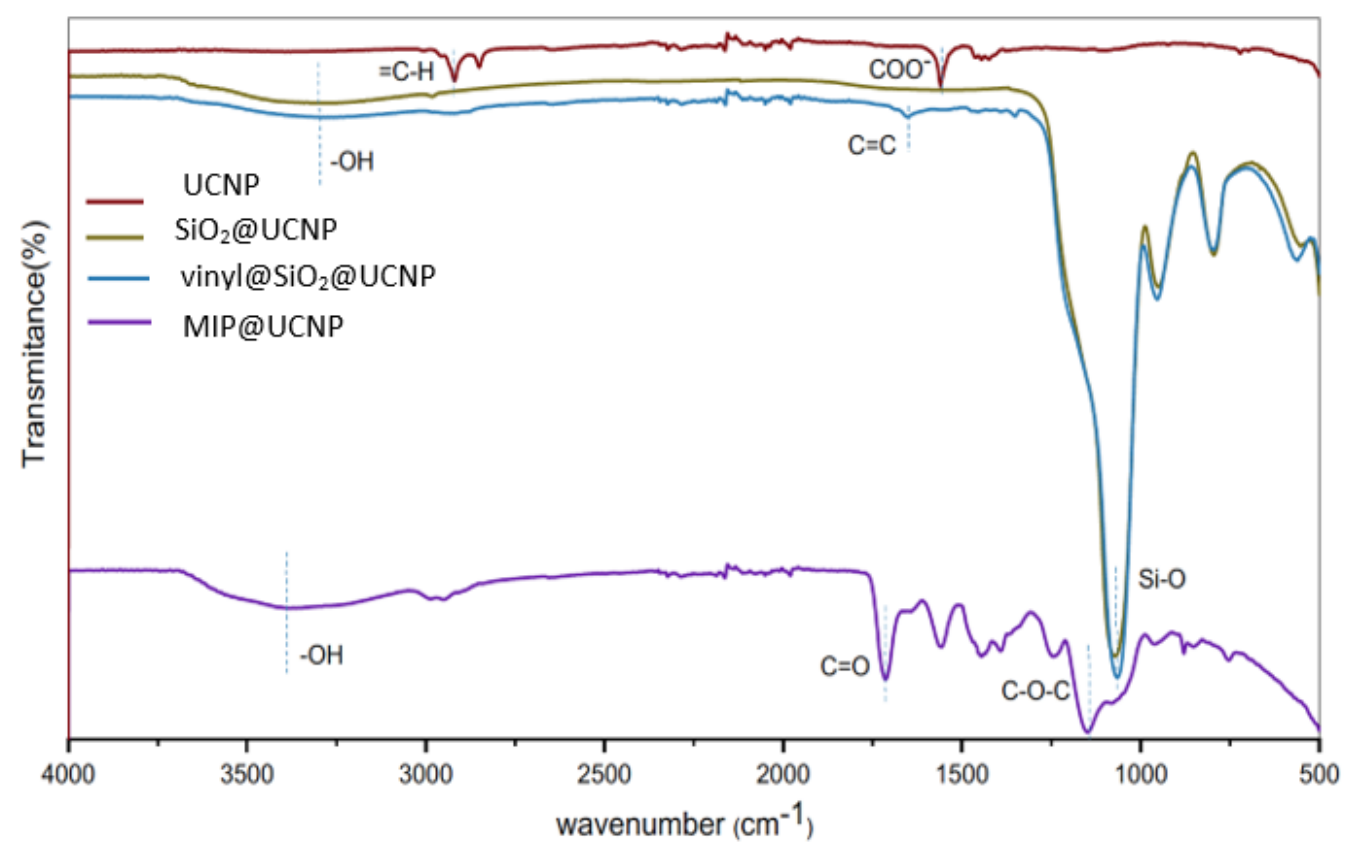

Figure 2. FTIR results of unmodified UCNPs and modified UCNPs and MIP @ UCNPs.

The application of MIP@UCNP nanoparticles in sensing the target molecule showed a great performance that will be discussed during the presentation

\section{Conclusions}

A novel synthesis method was advanced for the prepare of the MIP coated UCNPs to identification of a model dye (BR9) via RAFT polymerization. Six different method and three processes were used for the synthesis of the UCNPs and MIP coated UCNPs, respectively. Under optimal conditions, the MIPs showed good selectivity for the target dye against similar molecules and had a low LoD value, good integrity and sensitive for assign BR9 in aqueous solution. We repose that the results achieved are encouraging as they available major potential for the development of novel sensor platforms particular to several target molecules by carrying out similar experimental processes.

Acknowledgments: This work was kindly supported financially by the Scientific and Technological Research council of Turkey (TUBITAK) with a project number of $217 Z 069$.

\section{References}

1. Bagheri, A.; Arandiyan, H.; Boyer, C.; Lim, M. Lanthanide-doped upconversion nanoparticles: Emerging intelligent light-activated drug delivery systems. Adv. Sci. 2016, 3, 1500437.

2. $\mathrm{Wu}, \mathrm{S}$.; Butt, H.J. Near-infrared photochemistry at interfaces based on upconverting nanoparticles. Phys. Chem. Chem. Phys. 2017, 19, 23585-23596.

3. Li, L.; Hao, P.; Wei, P.; Fu, L.; Ai, X.; Zhang, J.; Zhou, J. DNA-assisted upconversion nanoplatform for imaging-guided synergistic therapy and laser-switchable drug detoxification. Biomaterials 2017, 136, 43-55. 
4. Wang, Y.; Ren, S.; Jiang, H.; Peng, Y.; Bai, J.; Li, Q.; Li, C.; Gao, Z.; Ning, B. A label-free detection of diethylstilbestrol based on molecularly imprinted polymer-coated upconversion nanoparticles obtained by surface grafting. RSC Adv. 2017, 7, 22215-22221.

5. Shan, S.N.; Wang, X.Y.; Jia, N.Q. Synthesis of NaYF 4: $\mathrm{Yb}^{3+}, \mathrm{Er}^{3+}$ upconversion nanoparticles in normal microemulsions. Nanoscale Res. Lett. 2011, 6, 1-5.

6. Liu, F.; Zhao, Q.; You, H.; Wang, Z. Synthesis of stable carboxy-terminated NaYF4: $\mathrm{Yb}^{3+}, \mathrm{Er}^{3+}$ @SiO2nanoparticles with ultrathin shell for biolabeling applications. Nanoscale 2013, 5, 1047-1053.

7. Huang, C.X.; Chen, H.J.; Li, F.; Wang, W.N.; Li, D.D.; Yang, X.Z.; Miao, Z.H.; Zha, Z.B.; Lu, Y.; Qian, H.S. Controlled synthesis of upconverting nanoparticles/CuS yolk-shell nanoparticles for: In vitro synergistic photothermal and photodynamic therapy of cancer cells. J. Mater. Chem. B 2017, 5, 9487-9496.

8. Muhr, V.; Wilhelm, S.; Hirsch, T.; Wolfbeis, O.S. Upconversion nanoparticles: From hydrophobic to hydrophilic surfaces. Acc. Chem. Res. 2014, 47, 3481-3493.

9. Wu, S.; Ning, Y.; Zhang, S. Hydrothermal Synthesis of $\beta$-NaYF4 :Yb, Er Nanocrystals with Upconversion Fluorescence Using Tetraethylene Pentamine as Chelating Ligand. J. Nanomater. 2012, 2012, 1-8.

10. Liu, B.; Chen, Y.; Li, C.; He, F.; Hou, Z.; Huang, S.; Zhu, H.; Chen, X.; Lin, J. Poly(Acrylic Acid) Modification of Nd3+-Sensitized Upconversion Nanophosphors for Highly Efficient UCL Imaging and pH-Responsive Drug Delivery. Adv. Funct. Mater. 2015, 25, 4717-4729.

11. Mondal, K.; Sharma, A. Recent advances in the synthesis and application of photocatalytic metal-metal oxide core-shell nanoparticles for environmental remediation and their recycling process. RSC Adv. 2016, 6, 83589-83612.

12. Spitale, A.; Perez, M.A.; Mejía-Rosales, S.; Yacamán, M.J.; Mariscal, M.M. Gold-palladium core@shell nanoalloys: Experiments and simulations. Phys. Chem. Chem. Phys. 2015, 17, 28060-28067.

13. Sekol, R.C.; Li, X.; Cohen, P.; Doubek, G.; Carmo, M.; Taylor, A.D. Silver palladium core-shell electrocatalyst supported on MWNTs for ORR in alkaline media. Appl. Catal. B Environ. 2013, 138-139, 285293.

14. Partyka, J.; Sitarz, M.; Leśniak, M.; Gasek, K.; Jeleń, P. The effect of SiO2/Al2O3 ratio on the structure and microstructure of the glazes from $\mathrm{SiO} 2-\mathrm{Al} 2 \mathrm{O} 3-\mathrm{CaO}-\mathrm{MgO}-\mathrm{Na} 2 \mathrm{O}-\mathrm{K} 2 \mathrm{O}$ system. Spectrochim. Acta Part A Mol. Biomol. Spectrosc. 2015, 134, 621-630.

15. Cachan, E.N.S.; Wilson, P.; Cachan, E.N.S.; Wilson, P.; Cnrs, C.; Urbain, G.; Iufm, F.; Mac, J. Synthesis and characterization of core-shell structure silica-coated Fe29. 5Ni70. 5 nanoparticles. Nanotechnology 2007, 18, $1-26$.

16. Yusof, N.A.; Rahman, S.K.A.; Hussein, M.Z.; Ibrahim, N.A. Preparation and characterization of molecularly imprinted polymer as SPE sorbent for melamine isolation. Polymers (Basel) 2013, 5, 1215-1228.

17. Liu, J.M.; Cao, F.Z.; Fang, G.Z.; Wang, S. Upconversion nanophosphor-involved molecularly imprinted fluorescent polymers for sensitive and specific recognition of sterigmatocystin. Polymers (Basel) 2017, 9, 299.

18. Tang, Y.; Li, M.; Gao, Z.; Liu, X.; Gao, X.; Ma, T.; Lu, X.; Li, J. Upconversion Nanoparticles Capped with Molecularly Imprinted Polymer as Fluorescence Probe for the Determination of Ractopamine in Water and Pork. Food Anal. Methods 2017, 10, 2964-2973.

19. Tang, Y.; Gao, Z.; Wang, S.; Gao, X.; Gao, J.; Ma, Y.; Liu, X.; Li, J. Upconversion particles coated with molecularly imprinted polymers as fluorescence probe for detection of clenbuterol. Biosens. Bioelectron. 2015, 71, 44-50.

20. Gwon, K.; Jo, E.J.; Sahu, A.; Lee, J.Y.; Kim, M.G.; Tae, G. Improved near infrared-mediated hydrogel formation using diacrylated Pluronic F127-coated upconversion nanoparticles. Mater. Sci. Eng. C 2018, 90, 77-84.

21. Zhu, K.; Liu, G.; Hu, J.; Liu, S. Near-Infrared Light-Activated Photochemical Internalization of ReductionResponsive Polyprodrug Vesicles for Synergistic Photodynamic Therapy and Chemotherapy. Biomacromolecules 2017, 18, 2571-2582.

22. Bagheri, A.; Arandiyan, H.; Adnan, N.N.M.; Boyer, C.; Lim, M. Controlled Direct Growth of Polymer Shell on Upconversion Nanoparticle Surface via Visible Light Regulated Polymerization. Macromolecules 2017, $50,7137-7147$.

23. Xie, Z.; Deng, X.; Liu, B.; Huang, S.; Ma, P.; Hou, Z.; Cheng, Z.; Lin, J.; Luan, S. Construction of Hierarchical Polymer Brushes on Upconversion Nanoparticles via NIR-Light-Initiated RAFT Polymerization. ACS Appl. Mater. Interfaces 2017, 9, 30414-30425. 
24. Cui, S.; Chen, H.; Gu, Y. Comparison of two strategies for the synthesis of upconverting nanoparticles as biological labels. J. Phys. Conf. Ser. 2011, 277, 012006.

25. Duong, H.T.T.; Chen, Y.; Tawfik, S.A.; Wen, S.; Parviz, M.; Shimoni, O.; Ab, D.J. Systematic investigation of functional ligands for colloidal stable upconversion nanoparticles. RSC Adv. 2018, 8, 4842-4849.

Publisher's Note: MDPI stays neutral with regard to jurisdictional claims in published maps and institutional affiliations.

(C) 2020 by the authors. Submitted for possible open access publication under the terms and conditions of the Creative Commons Attribution (CC BY) license (http://creativecommons.org/licenses/by/4.0/). 